\title{
Assessment of Turbulence-Chemistry Interaction Models in the National Combustion Code (NCC) - Part I
}

\author{
Thomas Wey ${ }^{1}$ and Nan-Suey Liu ${ }^{2}$ \\ NASA Glenn Research Center, OH 44135
}

\begin{abstract}
This paper describes the implementations of the linear-eddy model (LEM) and an Eulerian FDF/PDF model in the National Combustion Code (NCC) for the simulation of turbulent combustion. The impacts of these two models, along with the so called 'laminar chemistry' model, are then illustrated via the preliminary results from two combustion systems: a nineelement gas fueled combustor and a single-element liquid fueled combustor.
\end{abstract}

\section{Introduction}

Recent more stringent emission standards have enticed the development of more fuel-efficient and low-emission combustion system for aircraft gas turbine applications. The analysis and design of such advanced combustors is facilitated by employing higher fidelity, hence, more sophisticated turbulent combustion models. With advances in computational power and the availability of distributed computers, the use of complex turbulence-chemistry interaction models is now becoming more and more affordable for realistic geometries.

It is well known that the major difficulty, when modeling the turbulence-chemistry interaction, lies in the high non-linearity of the reaction rate expressed in terms of the temperature and species mass fractions. The so called 'laminar chemistry' model uses directly the averaged temperature and averaged species mass fractions to calculate the averaged reaction rate (which is, at the best, valid only for low turbulence flows). The transport probability density function ${ }^{1}$ (PDF) model and the linear eddy model $^{2}$ (LEM), both use local instantaneous values of the temperature and mass fractions, have been shown to often provide more accurate results of turbulent combustion. A filtered density function (FDF) is the spatial average of a PDF over a grid-box-sized volume ${ }^{3}$. The FDF has all the properties of a $\mathrm{PDF}^{4}$; i.e., FDF is non-negative everywhere and is normalized. The transport equation for the FDF/PDF and others like it has been widely studied by combustion engineers since the pioneering work of Lundgren $^{5,6}$. The linear eddy model (LEM) represents an alternate strategy in turbulent combustion. It is a onedimensional mixing model that combines molecular processes in a deterministic way (through the solution of unsteady reaction-diffusion equations), and turbulent transport through stochastic stirring events.

The main purposes of this report are to describe the practical implementations of the LEM and the FDF/PDF models, to illustrate the differences among the LEM, the transport FDF/PDF and the 'laminar chemistry' via the simulation results of a hydrogen gas fueled combustor, and to demonstrate their applications to a Jet-A liquid fueled combustor. These preliminary results will then lead to further, more quantitative investigations to be reported in the near future.

\section{Governing Equations for Gas-phase Flow}

The governing equations for the (averaged) flow in the multiple rotating frames of reference (MRF), which are convenient for including the rotational components of the jet engine in the computational domain ${ }^{7}$, are summarized in a hybrid formulation below.

Continuity equation:

\footnotetext{
${ }^{1}$ Aerospace Engineer, Combustion Branch, Member AIAA.

${ }^{2}$ Aerospace Engineer, Combustion Branch, Associate Fellow AIAA.
} 


$$
\frac{d}{d t} \iiint_{V} \rho d V+\iint_{A} \rho\left(\vec{u}-\vec{U}_{g}\right) \cdot d \vec{A}=\iiint_{V} S_{m l c} d V
$$

Species transport equation:

$$
\frac{d}{d t} \iiint_{V} \rho_{m} d V+\iint_{A} \rho_{m}\left(\vec{u}-\vec{U}_{g}\right) \cdot d \vec{A}=\iint_{A} \rho D_{e f f} \nabla\left(\frac{\rho_{m}}{\rho}\right) \cdot d \vec{A}+\iiint_{V} \dot{w}_{m}^{\prime \prime \prime} d V+\iiint_{V} S_{m l s} d V
$$

Momentum equation:

$$
\begin{aligned}
\frac{d}{d t} \iiint_{V} \rho \vec{u} d V & +\iint_{A} \rho \vec{u}\left(\vec{u}-\vec{U}_{g}\right) \cdot d \vec{A}=-\iint_{A} p d \vec{A}+\iint_{A} \tau \cdot d \vec{A} \\
& -\iiint_{V} \rho \vec{\omega}_{g} \times \vec{u} d V+\iiint_{V} S_{m l m} d V
\end{aligned}
$$

Energy equation:

$$
\begin{array}{r}
\frac{d}{d t} \iiint_{V} \rho E d V+\iint_{A} \rho E\left(\vec{u}-\vec{U}_{g}\right) \cdot d \vec{A}=-\iint_{A} p \vec{u} \cdot d \vec{A}-\iint_{A} \vec{q} \cdot d \vec{A} \\
\quad+\iint_{A} \vec{u} \cdot \tau \cdot d \vec{A}+\iiint_{V} \dot{q}^{\prime \prime} d V+\iiint_{V} S_{m l e} d V
\end{array}
$$

Alternate form of energy equation:

$$
\begin{gathered}
\frac{d}{d t} \iiint_{V} \rho C_{p} T d V+\iint_{A} \rho C_{p} T\left(\vec{u}-\vec{U}_{g}\right) \cdot d \vec{A}=\iiint_{V} \frac{D p}{D t} d V-\iint_{A} \vec{q} \cdot d \vec{A} \\
-\iint_{A} \sum_{m=1}^{N S} \rho m h_{m, \text { sens }} \overrightarrow{J_{m}} \cdot d \vec{A}-\iiint_{V} \sum_{m=1}^{N S} \dot{w}_{m}^{\prime \prime \prime}\left(\Delta h_{f}\right)_{m}^{o} d V+\iiint_{V} \dot{q}^{\prime \prime} d V \\
+\iint_{A} \vec{u} \cdot \tau \cdot d \vec{A}+\iiint_{V} S_{m l e} d V
\end{gathered}
$$

Turbulence equations:

$$
\begin{aligned}
& \frac{d}{d t} \iiint_{V} \rho k d V+\iint_{A} \rho k\left(\vec{u}-\vec{U}_{g}\right) \cdot d \vec{A}=S_{k}+\iint_{A}\left(\mu+\frac{\mu_{t}}{\sigma_{k}}\right) \nabla k \cdot d \vec{A} \\
& \frac{d}{d t} \iiint_{V} \rho \varepsilon d V+\iint_{A} \rho \varepsilon\left(\vec{u}-\vec{U}_{g}\right) \cdot d \vec{A}=S_{\varepsilon}+\iint_{A}\left(\mu+\frac{\mu_{t}}{\sigma_{\varepsilon}}\right) \nabla \varepsilon \cdot d \vec{A}
\end{aligned}
$$


$V$ is an arbitrary control volume with control surface $A$, and all the flow variables as well as the source terms including the reaction rates are in some averaged sense. $\rho$ is the fluid density, $\rho_{m}$ is the partial density of species $m, \dot{W}_{m}^{\prime \prime \prime}$ is the production rate of species $m$ per unit volume, $\vec{u}$ is the flow velocity in a stationary Cartesian coordinate system, $\vec{\omega}_{g}$ is the grid rotating velocity for each designated axis, $\vec{U}_{g}=\vec{\omega}_{g} \times \vec{r}, E$ is the total energy density, $k$ is the turbulent kinetic energy, $\varepsilon$ is the turbulent dissipation rate, $S_{k}$ is the source term for $k, S_{\varepsilon}$ is the source term for $\varepsilon, p$ is the static pressure, $\tau$ is the effective stress tensor, $\vec{q}$ is the effective heat flux vector, $D_{\text {eff }}$ is the effective mass diffusivity coefficient which is equal to $\frac{\mu_{t}+\mu}{\rho S c}, \mu$ and $\mu_{t}$ are the laminar viscosity and turbulent viscosity, respectively. $S_{m l c}, S_{m l s}, S_{m l m}$ and $S_{m l e}$ are source terms due to the contribution of spray, respectively. For gas, the Schmidt number $(S C$ ) is about 1. Different angular velocities (and even different rotating axes) are assigned to different mesh blocks or groups within the model. Balance equations for each group are expressed in the relative reference frame but in terms of the absolute velocity (i.e. the velocity with respect to a stationary coordinate system).

\section{Turbulence-Chemistry Interaction}

A rudimentary model for the turbulence-chemistry interaction is the so called 'laminar chemistry' model, which directly uses the averaged temperature and averaged species mass fraction to calculate the averaged reaction rates. Due to the high non-linearity of the reaction rates with respect to the temperature and species mass fractions, the laminar chemistry model is, at the best, valid only for low turbulence flows. This motivates the use of much more sophisticated models such as the FDF/PDF model and the LEM model.

\section{Linear-Eddy Model}

The linear-eddy model was proposed by Kerstein ${ }^{8}$ for non-reacting flow and extended to reactive flow ${ }^{9}$. It has been investigated in detail in e.g. Reference [2], therefore, only a briefly summary is given here. The LEM approach accounts for both deterministic and stochastic contributions. The effects of the unresolved part of the velocity on the momentum and scalar transport are explicitly represented by turbulent stirring events. The LEM model is implemented in terms of a fractional splitting technique; it is divided into two processes: subgrid and supergrid. The subgrid process, which occurs within each computational cell, composes of four operators: (a) molecular diffusion, (b) finite-rate kinetics, (c) volumetric heat release, and (d) stochastic stirring that actually is a turbulent convection by a unresolved velocity component. The supergrid process represents the convection of the scalar field by the resolved velocity field. The subgrid process is achieved by first subdividing each cell into a fixed number of lumps, then the lumps are aligned from inflow to outflow within a cell forming a one dimensional path. The subgrid LEM governing equations are solved on each LEM lump.

\section{Subgrid processes:}

Species transport equation for LEM model:

$$
\frac{d Y_{i}}{d t}=\frac{\dot{w}_{i}^{\prime \prime \prime}}{\rho_{\ell}}+\nabla \cdot\left(\frac{v}{S c} \frac{d Y_{i}}{d \ell}\right)+F_{Y_{i}, \text { stirring }}+S_{m l s}
$$

Energy equation::

$$
\frac{d T_{\ell}}{d t}=-\frac{1}{C p_{\ell}} \sum_{i}^{N S} \dot{w}_{m}^{\prime \prime \prime}\left(\Delta h_{f}\right)_{i}^{o}+\frac{1}{C p_{\ell}} \frac{d}{d \ell}\left(\sum_{i}^{N S} \frac{v_{\ell}}{S c} \frac{d Y_{i}}{d \ell} C p_{i} T\right)+\frac{1}{\rho C p_{\ell}} \frac{d}{d \ell}\left(k_{\ell} \frac{d T}{d \ell}\right)+F_{T, \text { stirring }}+S_{m l e}
$$


$F_{T, \text { stirring }}$ and $F_{Y_{i} \text {,stirring }}$ are the unresolved turbulent convective terms and implemented explicitly using the stochastic re-arrangement events called triplet maps. The triplet map mimics the characteristics of the stretching and folding processes inherent to turbulent flows. The effect of a triplet map is a three-fold compression of the scalar field in a typical eddy size, $l$. The map is parameterized by the length-scale of a notional "eddy" acting on the lumps, $\ell$, the starting position in the lumps and the stirring frequency, $\lambda$. For convenience, the position is randomly taken to indicate the starting location of the eddy from a uniform distribution. The eddy size is randomly selected from a prescribed distribution function given by ${ }^{8}$

$$
f(l)=(5 / 3) l^{-8 / 3} /\left[\eta^{-5 / 3}-l_{t}^{-5 / 3}\right]
$$

Here the Kolmogorov scale $\eta$ is determined from inertial range scaling law, $\eta \sim l_{t} \operatorname{Re}^{-3 / 4}, l_{t}$ is turbulence length scale. The event rate (frequency per unit length) is determined by ${ }^{8}$

$$
\lambda=\frac{54}{5} \frac{v \operatorname{Re}_{t}}{C_{\lambda} l_{t}^{3}} \frac{\left[\left(l_{t} / \eta\right)^{5 / 3}-1\right]}{\left[1-\left(\eta / l_{t}\right)^{4 / 3}\right]}
$$

The model constant $C_{\lambda}$ is 15 . The stirring time interval between events is give as $\Delta t_{\text {stir }}=1 /(\lambda \Delta)$ where $\Delta$ is the total length of the lumps which is volume ${ }^{1 / 3}$ of a cell.

Supergrid processes:

Lagrangian convection (also known as splicing)

$$
\frac{\partial \phi}{\partial t}=-\nabla \cdot\left(\vec{u}-\vec{U}_{g}\right)
$$

where $\phi$ is $Y_{i}$ or $T$. This equation describes the mesh-resolved convection of the scalar field, and is solved via a Lagrangian transfer of mass across the finite-volume cell surfaces ${ }^{10,11}$.

Referring to Figure 1, for example, the outward mass through the right side of a cell computed from resolved velocity and density is equivalent to 1.5 LEM lumps and colored in red. The outward mass through the bottom side of the mesh is equivalent to 2.5 LEM lumps and colored in magenta. Similarly the inward mass through the top and left sides of the mesh are equivalent to 6 LEM lumps that are added to existing lumps. The net number of lumps is 14 after the splicing step. It leads to a non-uniform number of lumps in each mesh. To avoid programming complexities in a parallel environment, the LEM domain is re-gridded to have lumps of equal volume and equal number after each splicing. 


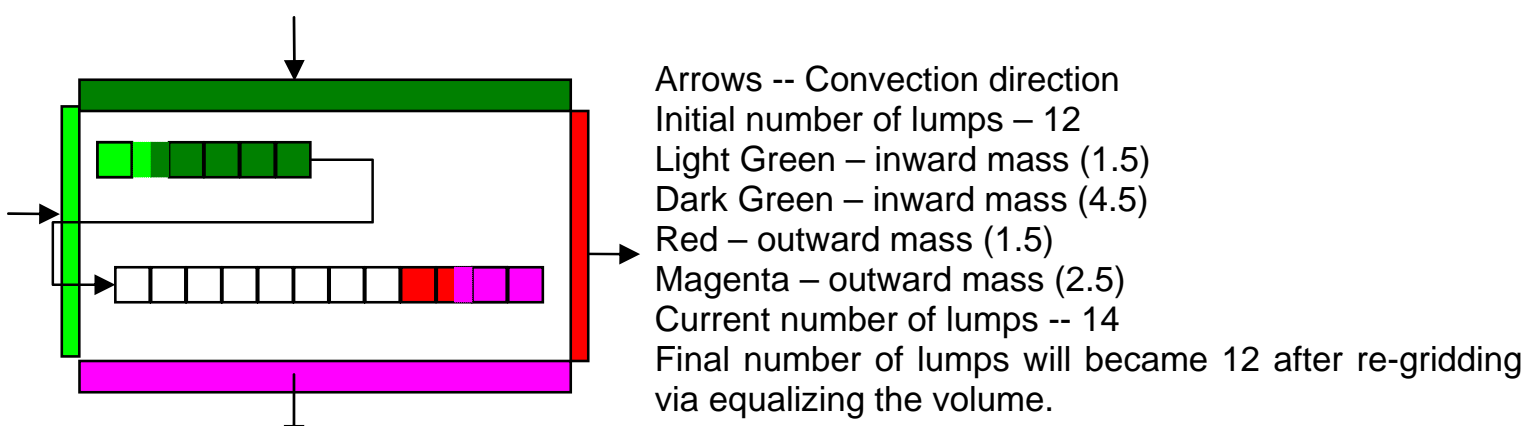

Figure 1 Schematic illustrating the splicing algorithm used for scalar convection. (Assuming number of LEM lumps is 12. Each lump contains volume and species density)

Some salient features of the LEM module are summarized below:

1. The LEM code receives the mean velocity, pressure and turbulence fields from the flow solver. And, also, it receives the source terms arising from the liquid-phase contribution from the spray solver if needed.

2. The LEM solver provides the species and temperature solution to the flow solver and, also, to the spray solver if needed.

3. The LEM model treats the nonlinear reaction rates without any approximation.

4. The diffusion term in the subgrid process puts constraints on time step and usually requires multiple subiterations.

\section{FDF/PDF model}

The Eulerian joint scalar filtered mass-density function (FDF) approach for unsteady flow or the Eulerian joint scalar probability density function (PDF) for steady flow has been discussed in detail in the literature ${ }^{1,12,13}$ and , therefore is only briefly summarized here. Like LEM mode, the transport equation of the probability density function is solved by making use of an approximate factorization scheme. It is split into four operators associated with chemical reactions, molecular mixing, spray and spatial transport. It is solved by making use of a Monte Carlo technique. In the Monte Carlo simulation the density function is represented by a large ensemble of stochastic particles. Each particle carries enthalpy, temperature and species mass fraction

\section{Subgrid processes:}

Species transport equation for FDF/PDF model

$$
\frac{d Y_{i}}{d t}=\frac{\dot{w}_{i}^{\prime \prime \prime}}{\rho_{\ell}}-C_{\phi} \omega\left(Y_{i}-\bar{Y}_{i}\right)+S_{m l s}
$$

Energy equation

$$
\frac{d h_{\ell}}{d t}=-C_{\phi} \omega\left(h_{\ell}-\bar{h}\right)+S_{m l e}
$$

Where $\omega=\varepsilon / k, C_{\phi}$ is an empirical constant with a value of about one. The first term on the right hand side of the energy equation represents molecular mixing which is accounted for by making use of the relaxation to the ensemble mean submodel ${ }^{14}$. The last term, $S_{m l e}$, represents the contribution from the spray source terms.

\section{Supergid processes:}


Eulerian convection:

$$
\frac{\partial \phi}{\partial t}=-\frac{1}{\rho} \nabla \cdot\left[\rho\left(\vec{u}-\vec{U}_{g}\right)+\frac{\mu_{e}}{S c_{\phi}} \nabla \phi\right]
$$

where $\phi$ is $Y_{i}$ or $h_{\ell}, \mu_{e}$ is effective viscosity, $S c_{\phi}$ is Schmidt number of $\phi, \rho$ is the mixture density in the cell.

Referring to Figure 2, unlike the LEM splicing algorithm, the Eulerian convection and diffusion process is achieved through the content replacement of particles, as each Monte Carlo particle carries the information of the mass fraction of species, the enthalpy, the temperature, but not the volume. For example, the net inward mass through the right side of a cell, computed from upwind-resolved convective terms and central-differenced effective diffusion term; then normalized by the mass in the cell, is equal to $5 \%$ and colored in red. The net inward mass through the bottom side of the mesh is equal to $10 \%$ and colored in magenta. Similarly the inward mass through the top and left sides of the mesh are equivalent to $40 \%$ and colored in dark and light green. If the total number of the particles is 20 in this cell, then one particle (5\% of 20 ) will be randomly selected from its right adjacent cell, and its contents are copied to one randomly selected particle in the current cell. The same procedure is applied to other particles in colors. For those remaining particles ( $45 \%$ here) the contents are randomly shuffled within the cell. This is equivalent to an upwind differencing scheme.
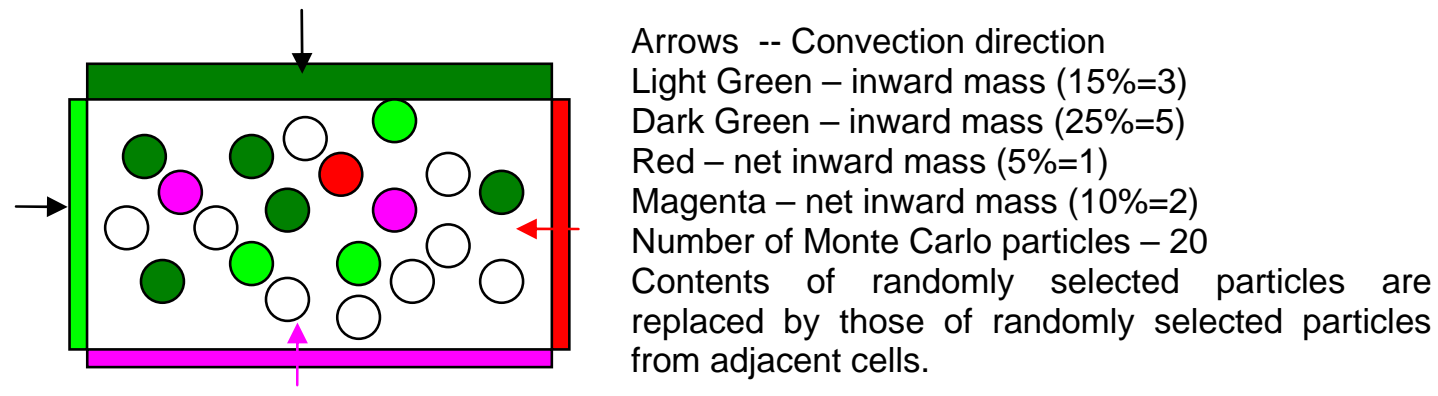

\section{Figure 2. Schematic illustrating the process of the scalar convection and diffusion for the Eulerian scalar} Monte Carlo FDF/PDF model.

Some salient features of the FDF/PDF module are summarized below:

1. The FDF/PDF code receives the mean velocity, pressure and turbulence fields from the flow solver. And, also, it receives the source terms arising from the liquid-phase contribution from the spray solver if needed.

2. The FDF/PDF solver provides the species and temperature solution to the flow solver and, also, to the spray solver if needed.

3. The FDF/PDF model treats the nonlinear reaction rates without any approximation.

Finally, all the operators of LEM or FDF/PDF are integrated by an explicit Euler method.

\section{Preliminary Results}

The effects of employing different turbulence-chemistry models for turbulent combustion are illustrated by the calculated results from the simulations of two combustors: a Sandia 9-element hydrogen combustor and a NASA single-element lean direct injection combustor. 


\section{Sandia 9-element hydrogen gas fueled combustor}

The simulation of the hydrogen burner involved two separate computations. First a simulation of just one nozzle was performed, which included an upstream settling chamber, and a downstream expansion. The size of the settling chamber and expansion chamber was made to provide an identical expansion of area as that of the nine-nozzle case. The two hydrogen jets were provided with plenums, to damp out any possible fluctuations of the inlet conditions.

After the simulation of the single nozzle case had run to convergence, data was taken at a location $.005 \mathrm{~m}$. upstream of the expansion region. This data is just downstream of the hydrogen jets, and is used as the initial conditions for the nine-nozzle simulation.

The quantities recorded at this location are the distributions of the velocity, turbulence and scalar variables over a 2D area. These fields are then interpolated onto the inlet regions of the nine-element simulation. Each of the nine nozzles had the initial data so orientated that the hydrogen jets corresponded to the experimental location. The grid used contained 282,624 hexahedrons and is shown in Fig. 3. The chemical kinetics is represented by a 9-species, 20step mechanism for hydrogen-air combustion.
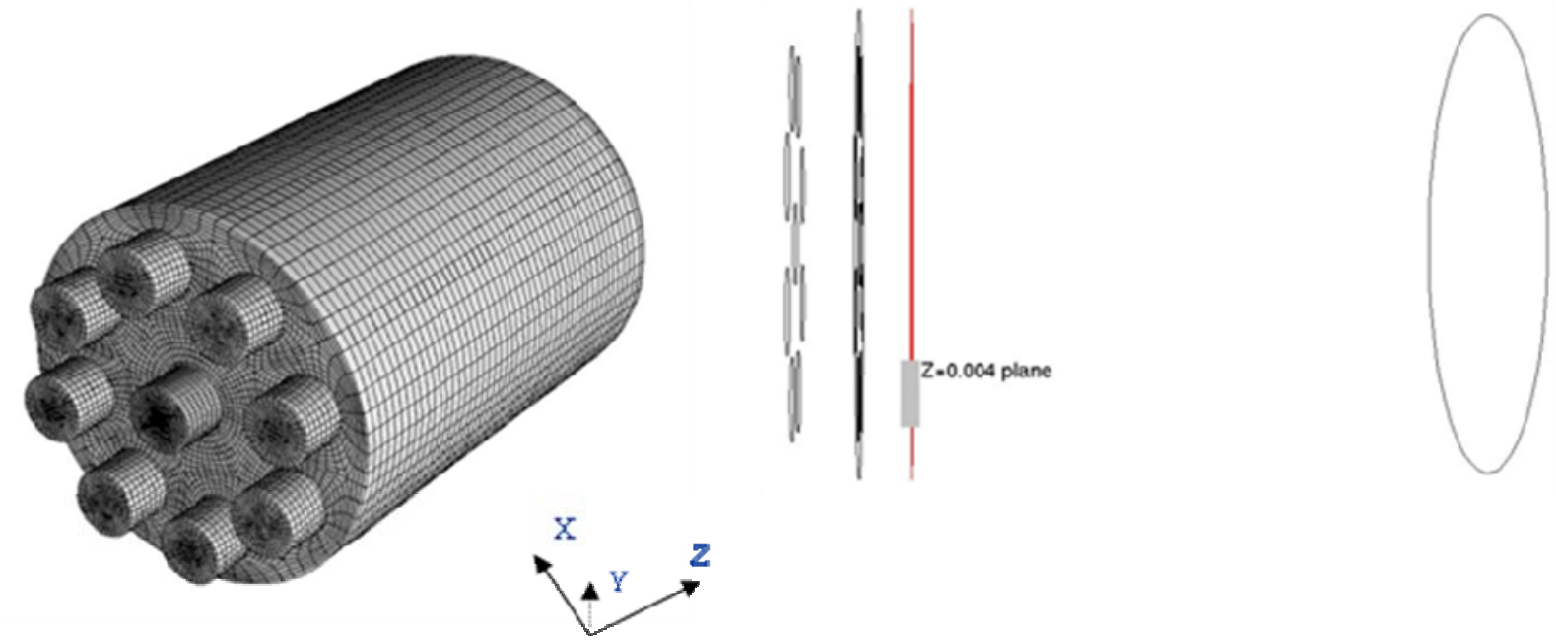

Figure 3. Schematic of the mesh used in the simulation of a Sandia 9-element hydrogen burner. It consists of 282624 hexahedrons. Length is about 0.05 meter and diameter is about 0.038 meter. Each injector is 0.076 meter in diameter.

Computed axial velocity on the vertical center plane ( $\mathrm{x}=0.0$ plane) by using different turbulent combustion models are presented in Figure 4. The top two plots are results of URANS simulations while the bottom three plots are results of RANS simulations. It can be seen that the pattern of axial velocity from URANS and RANS is quite different. Figure 5 shows the axial velocity on $\mathrm{z}=0.004 \mathrm{~m}$ plane viewed from the exit. It indicates that results between FDF/PDF and laminar chemistry are quite different too. Figure 6 presents the pressure contours on $\mathrm{x}=-$ $0.0014 \mathrm{~m}$.

The temperature distribution on $\mathrm{z}=0.004 \mathrm{~m}$ is depicted in Figure 7 . The results from the laminar chemistry model are distinctly different from those from the FDF/PDF model. Figure 8 shows that RANS simulations yield lower temperature flows from the inlet of the central injector all the way to the exit of the combustion chamber, but this is not the case for URANS simulations. The mass fraction of $\mathrm{OH}$, a minor species, is presented in Figure 9 ( $\mathrm{z}=0.004 \mathrm{~m}$ plane) and Figure 10 ( $x=0.0 \mathrm{~m}$ plane). Basically, it reflects the structure of the temperature. 

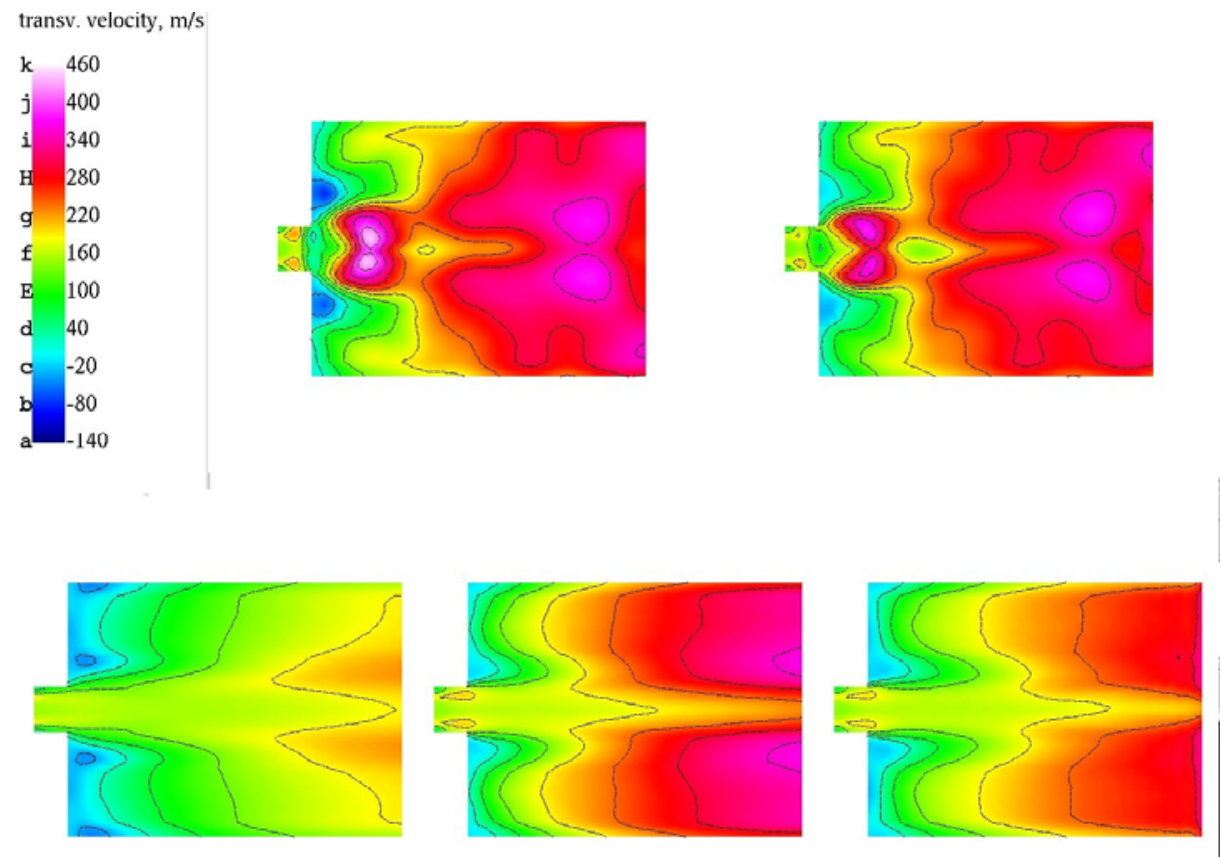

Figure 4. Axial velocity contours at $\mathrm{x}=\mathbf{0 . 0} \mathrm{m}$ plane. Plots in clockwise direction: FDF/URANS with steady pressure exit; FDF/URANS with unsteady pressure exit; PDF/RANS with sensible enthalpy correction; PDF/RANS; and laminar chemistry/RANS.
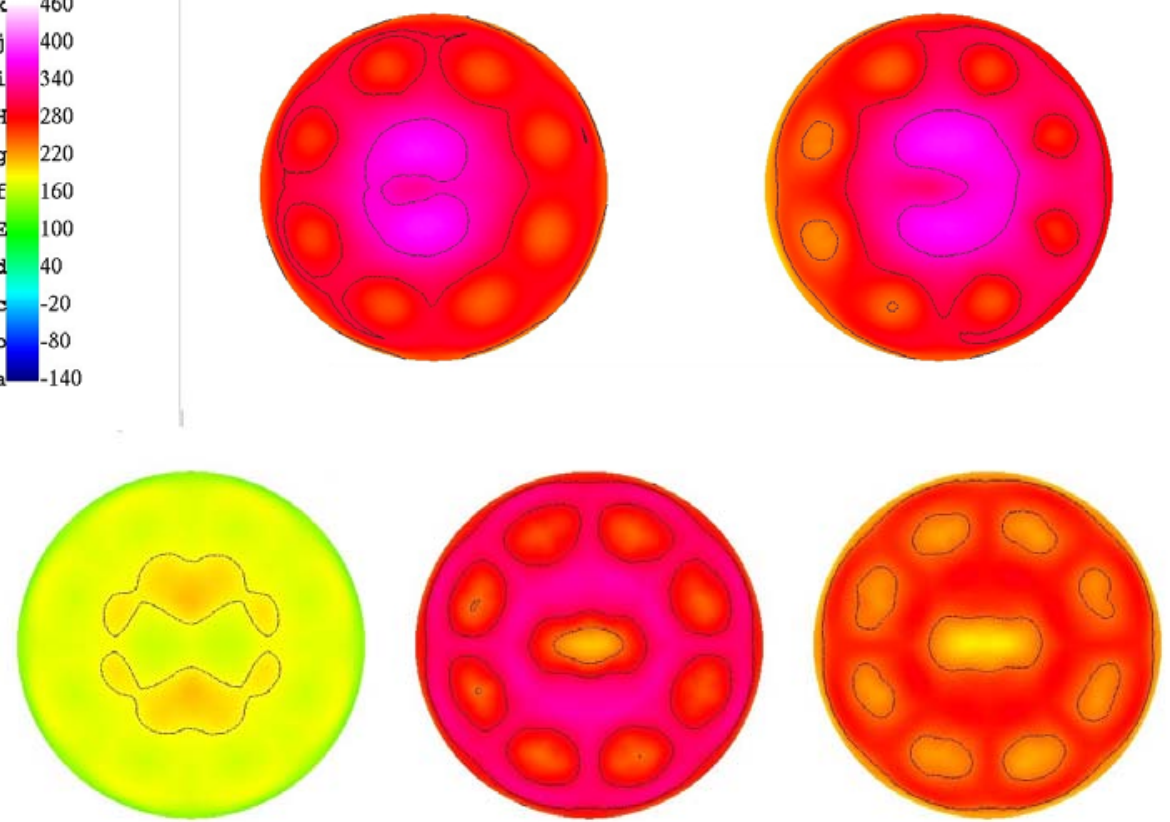

Figure 5. Axial velocity contours at $\mathrm{z}=\mathbf{0 . 0 0 4} \mathrm{m}$ plane. Plots in clockwise direction: FDF/URANS with steady pressure exit; FDF/URANS with unsteady pressure exit; PDF/RANS with sensible enthalpy correction; PDF/RANS; and laminar chemistry/RANS. 

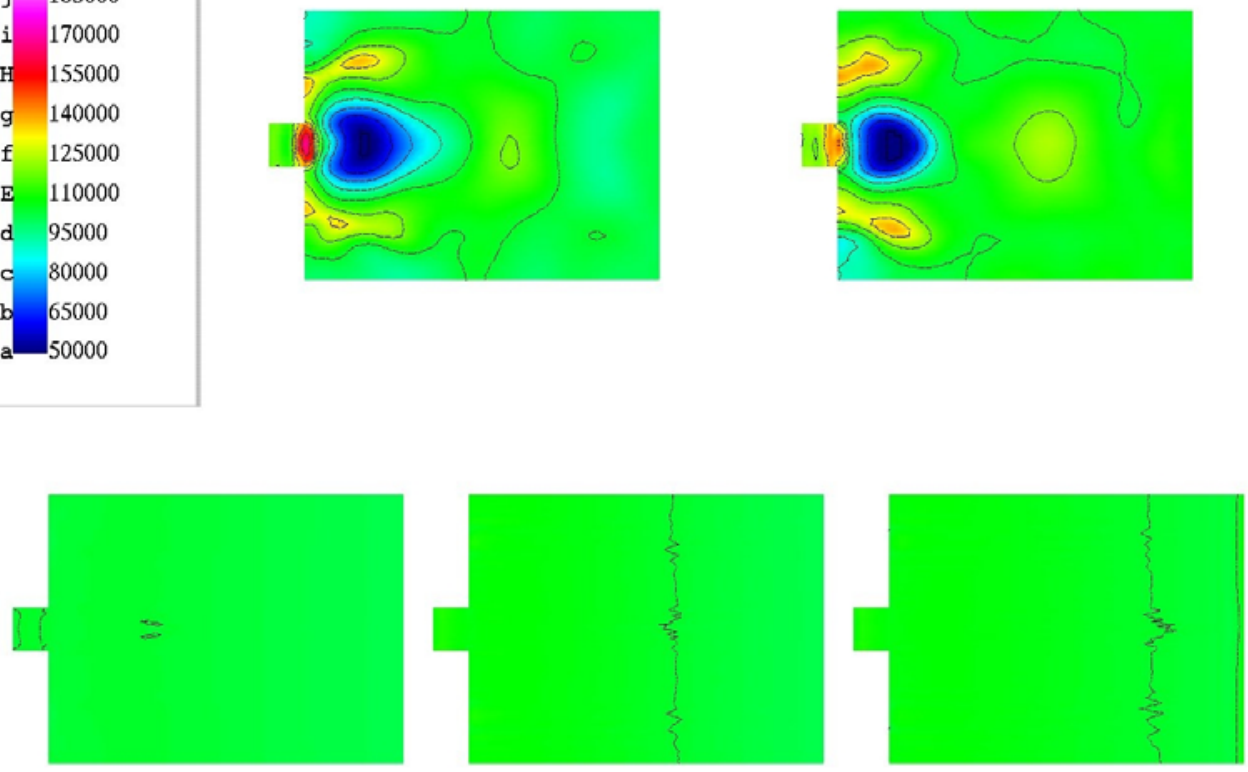

Figure 6. Pressure contours at $\mathrm{x}=0.0014 \mathrm{~m}$ plane. Plots in clockwise direction: FDF/URANS with steady pressure exit; FDF/URANS with unsteady pressure exit; PDF/RANS with sensible enthalpy correction; PDF/RANS; and laminar chemistry/RANS.

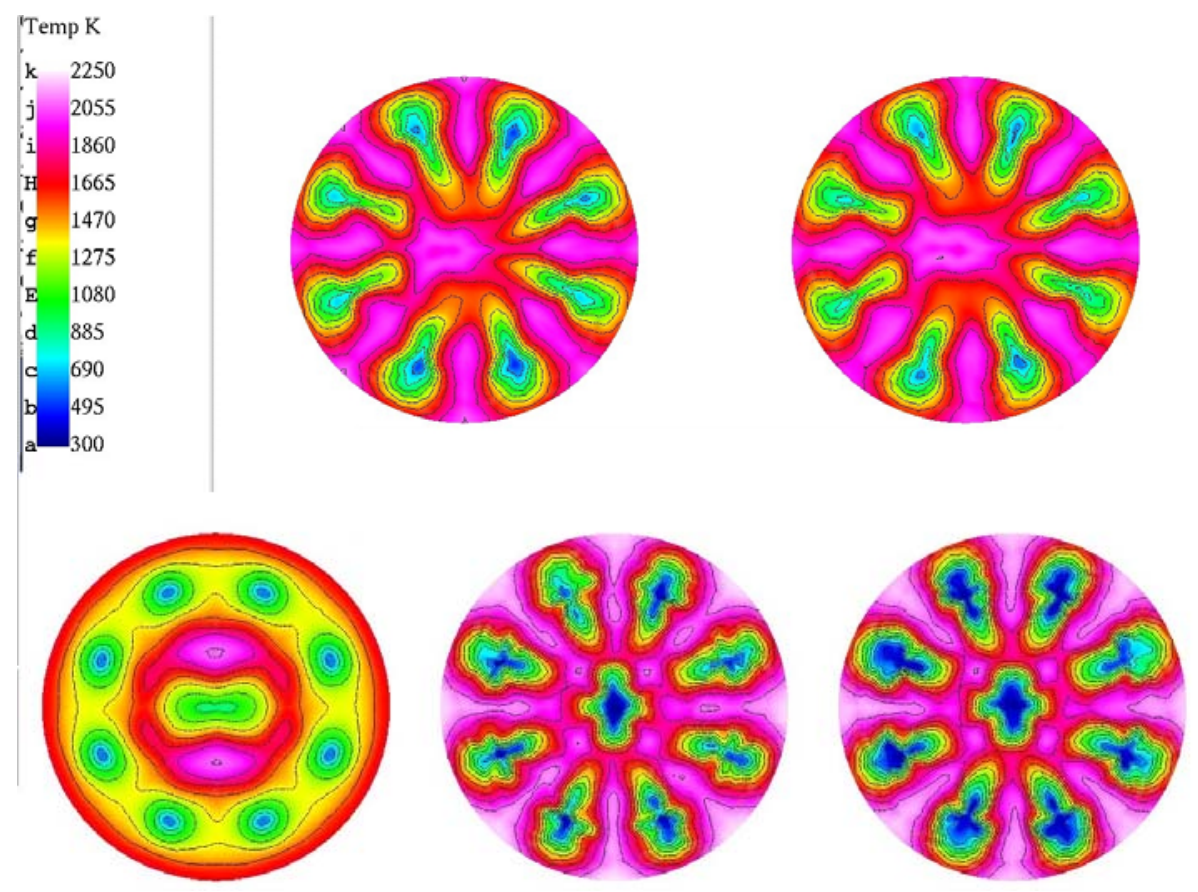

Figure 7. Temperature contours at $\mathrm{z}=\mathbf{0 . 0 0 4} \mathrm{m}$ plane. Plots in clockwise direction: FDF/URANS with steady pressure exit; FDF/URANS with unsteady pressure exit; PDF/RANS with sensible enthalpy correction; PDF/RANS; and laminar chemistry/RANS. 

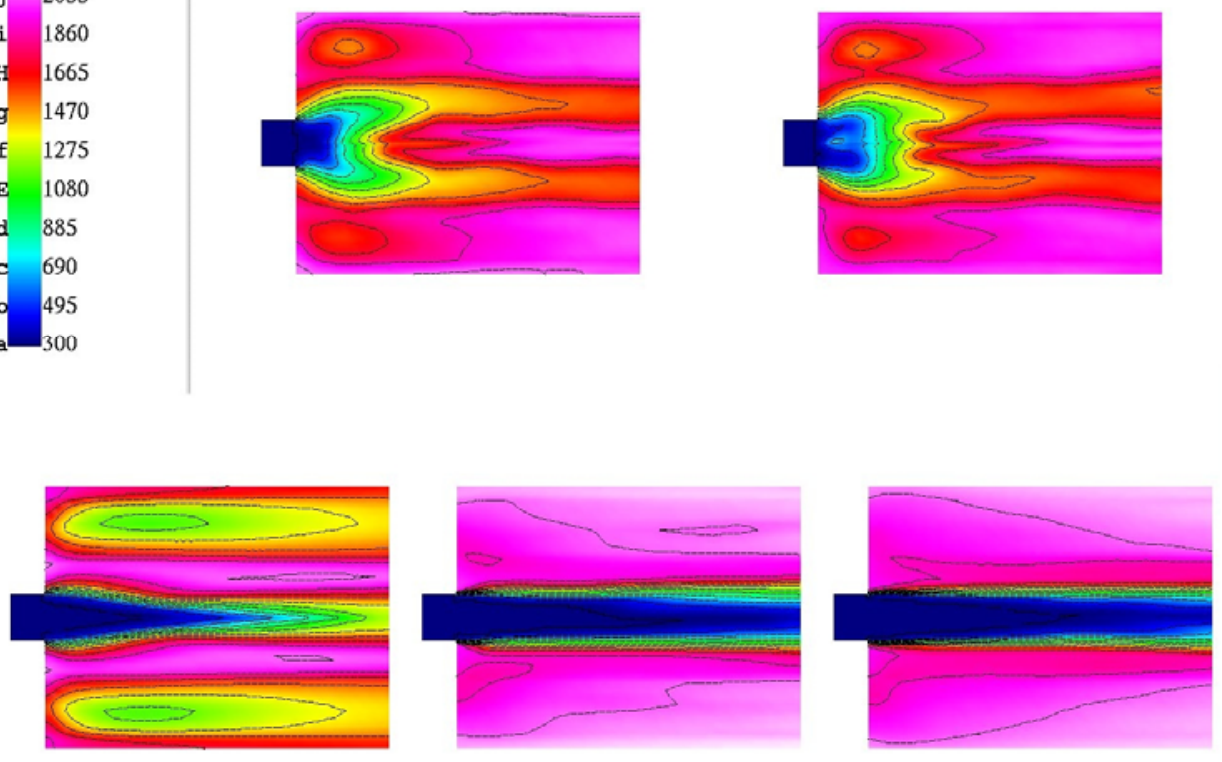

Figure 8. Temperature contours at $\mathrm{x}=0.0 \mathrm{~m}$ plane. Plots in clockwise direction: FDF/URANS with steady pressure exit; FDF/URANS with unsteady pressure exit; PDF/RANS with sensible enthalpy correction; PDF/RANS; and laminar chemistry/RANS.

$$
\begin{array}{ll}
\multicolumn{2}{c}{\mathrm{OH} \mathrm{kg} / \mathrm{kg}} \\
\mathrm{k} & 1.200000 \mathrm{e}-02 \\
\mathrm{j} & 1.080000 \mathrm{e}-02 \\
\mathrm{i} & 9.600000 \mathrm{e}-03 \\
\mathrm{H} & 8.400000 \mathrm{e}-03 \\
\mathrm{~g} & 7.200000 \mathrm{e}-03 \\
\mathrm{f} & 6.000000 \mathrm{e}-03 \\
\mathrm{E} & 4.800000 \mathrm{e}-03 \\
\mathrm{~d} & 3.600000 \mathrm{e}-03 \\
\mathrm{c} & 2.400000 \mathrm{e}-03 \\
\mathrm{~b} & 1.200000 \mathrm{e}-03 \\
\mathrm{a} & 0.000000 \mathrm{c}+00
\end{array}
$$
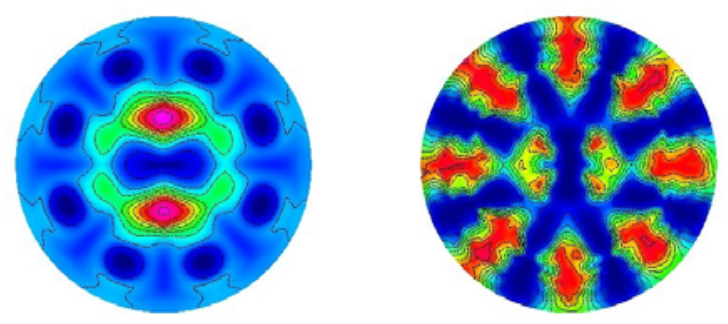
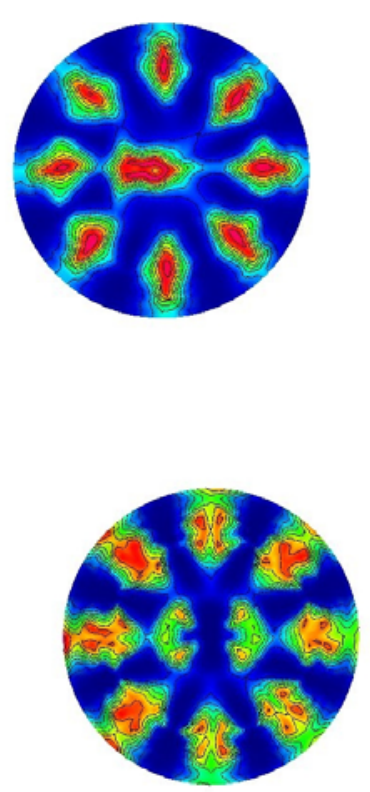

Figure 9. Species $\mathrm{OH}$ mass fraction contours at $\mathrm{z}=\mathbf{0 . 0 0 4} \mathrm{m}$ plane. Plots in clockwise direction: FDF/URANS with steady pressure exit; FDF/URANS with unsteady pressure exit; PDF/RANS with sensible enthalpy correction; PDF/RANS; and laminar chemistry/RANS. 

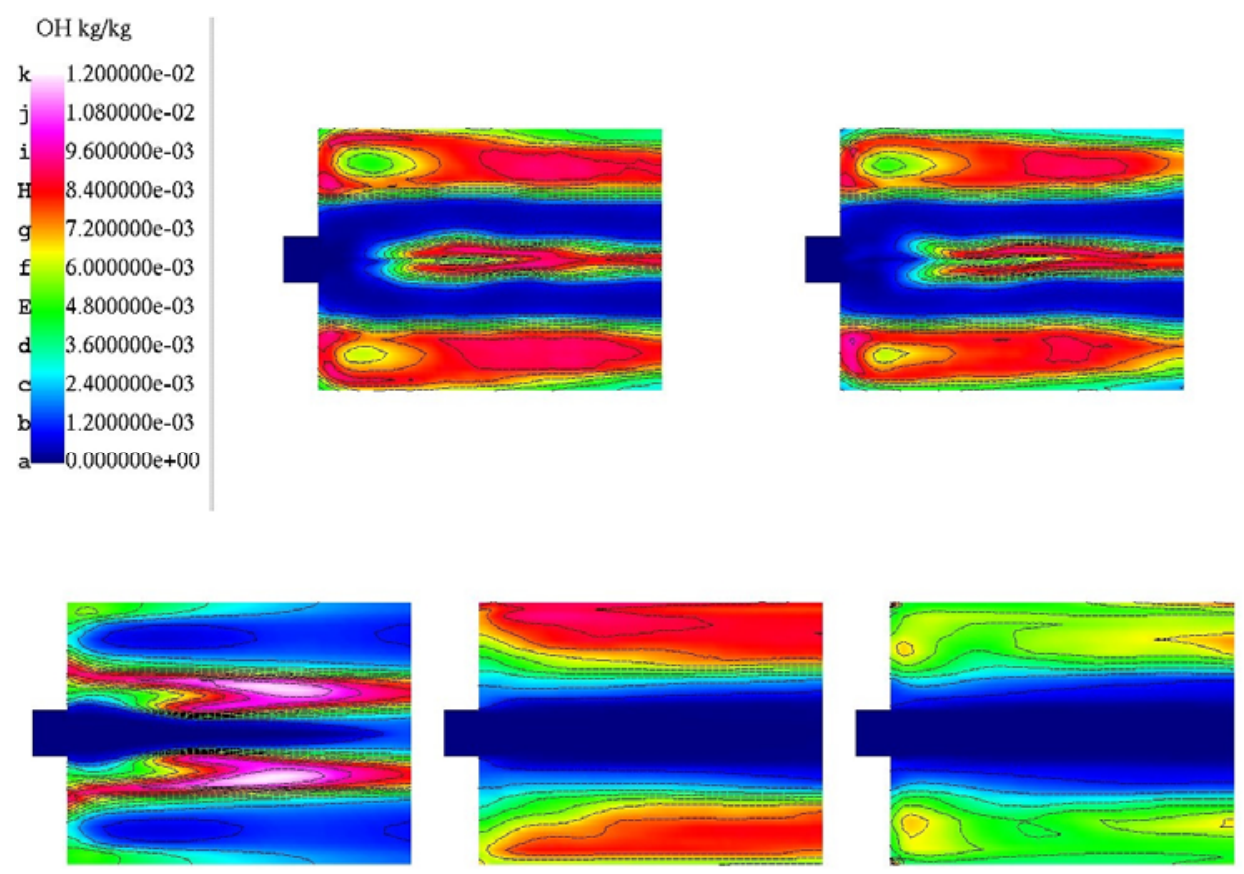

Figure 10. Species $\mathrm{OH}$ mass fraction contours at $\mathrm{x}=\mathbf{0 . 0} \mathrm{m}$ plane. Plots in clockwise direction: FDF/URANS with steady pressure exit; FDF/URANS with unsteady pressure exit; PDF/RANS with sensible enthalpy correction; PDF/RANS; and laminar chemistry/RANS.

So far, we have presented the results obtained in the context of RANS and URANS simulations. In the following, the results obtained in the context of the partially resolved numerical simulation for a very large eddy simulation (PRNS/VLES) ${ }^{15,16,17}$ will be presented. The PRNS/VLES is based on the concept of temporal filtering, in which the contents of both the resolved and unresolved turbulence are regulated by a "resolution control parameter" (RCP), which is related to the width of the temporal filter. A value of RCP $=0.4$ is used in the following cases.

While the PRNS is used to solve the pressure (i.e. the continuity equation), the momentum and the turbulence equations, the temperature (or enthalpy) and the species mass fraction are solved either via the FDF or the linear eddy model (LEM) approach. For sub-grid scalar field evolution, 24 LEM cells are used within each PRNS cell, allowing a resolution in the sub-grid approximately of the order of $\eta$, the Kolmogorov scale. For the scalar FDF simulation, 100 particles were used in each PRNS cell to represent the joint scalar FDF.

In Figure 11, several isosurface plots of the axial velocity computed by LEM/PRNS are shown. It indicates that the center injector has the largest penetration. The distribution of the $\mathrm{OH}$ mass fraction is depicted in Figure 12. 


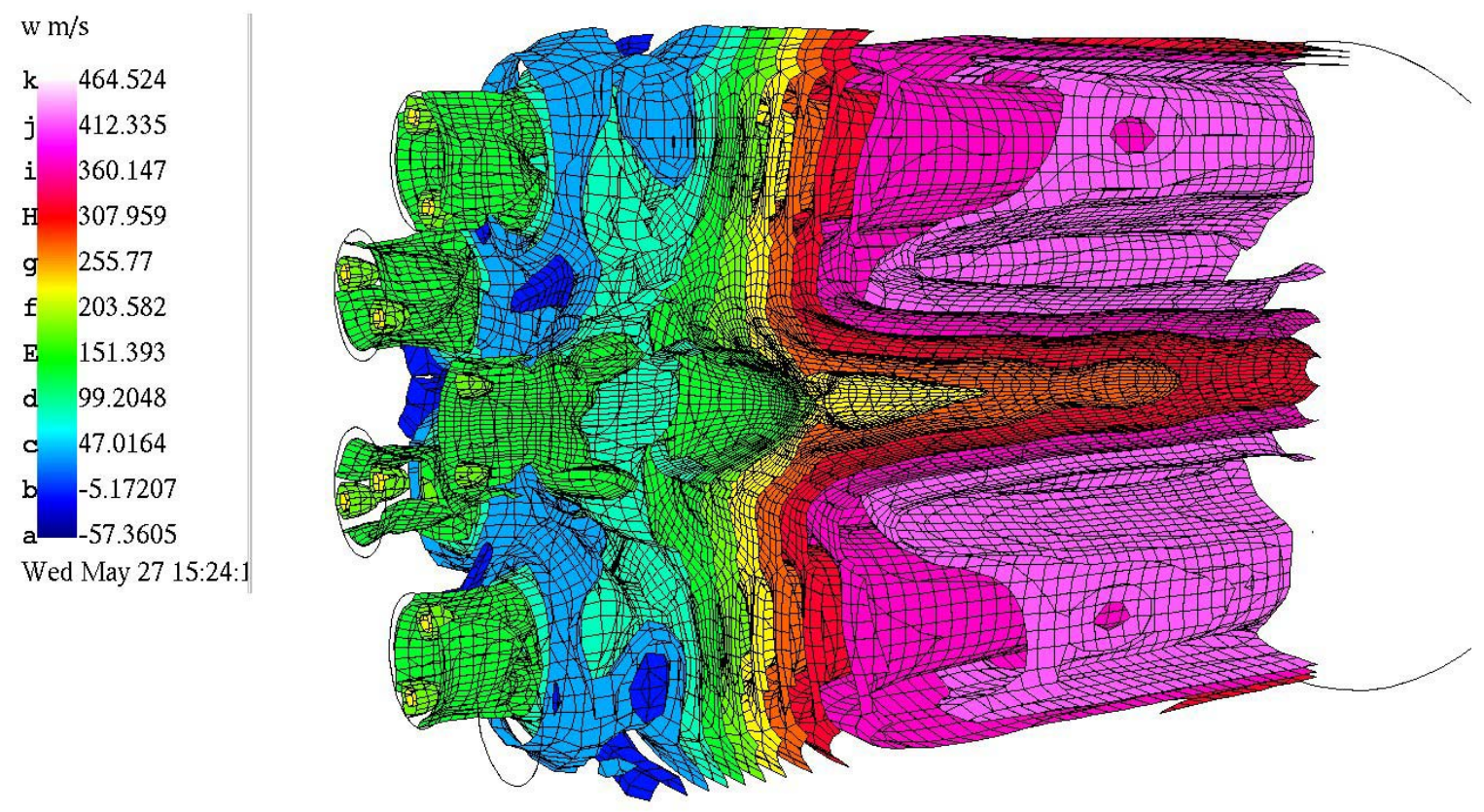

Figure 11. Isosurfaces of the axial velocity (partial view). LEM/PRNS with unsteady pressure exit.

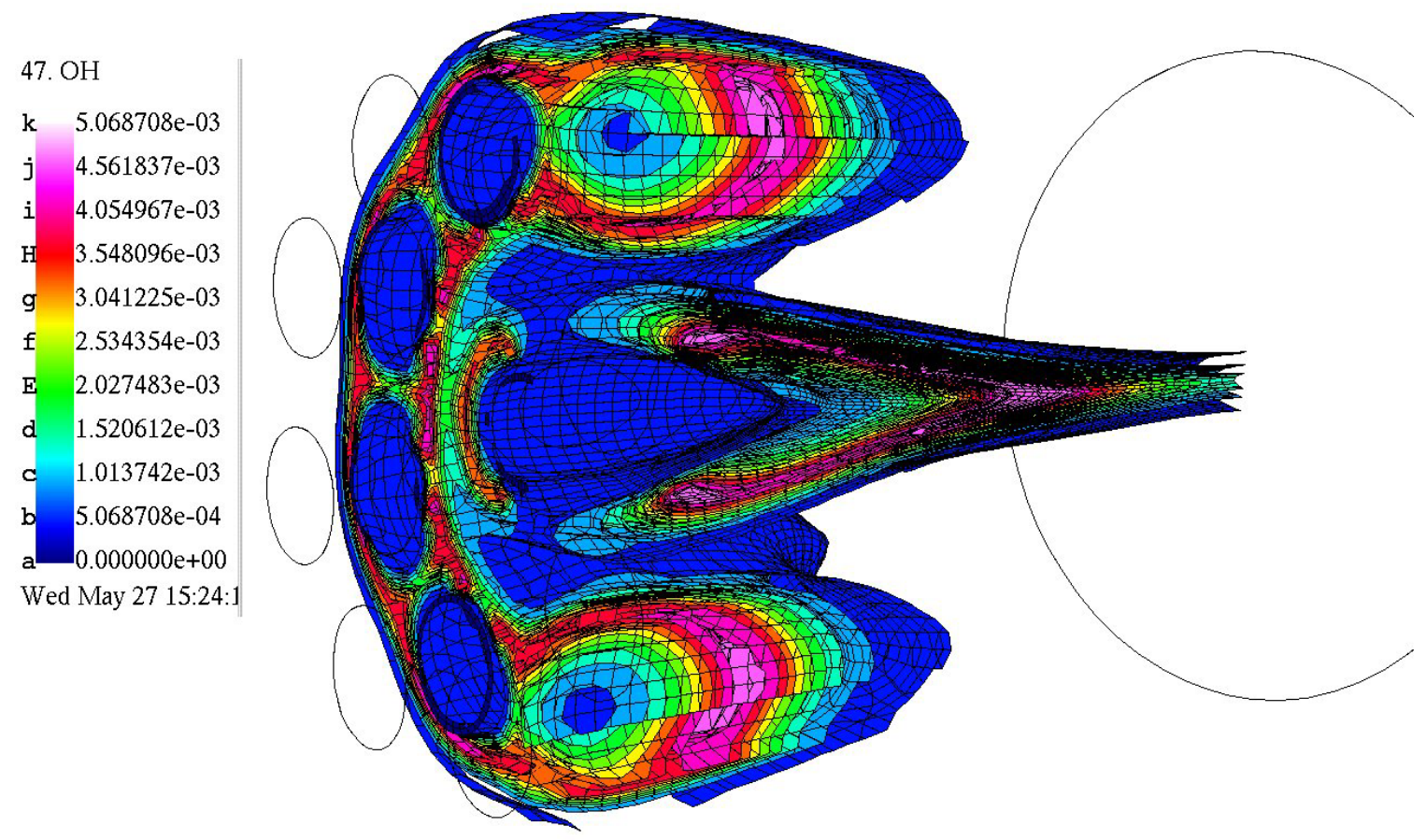

Figure 12. Isosurfaces of the $\mathrm{OH}$ mass fraction (partial view). LEM/PRNS with unsteady pressure exit. 
Selected results from the FDF/PRNS and the LEM/PRNS calculations are shown in Figure 13 to 15. Figure 13 shows the isosurface of axial velocity at $200 \mathrm{~m} / \mathrm{s}$ by FDF (top plot) and by LEM (bottom plot) approaches. It suggests that the flow field from LEM is more organized than that from FDF. Figure 14 depicts the isosurface of temperature at 2000K. It suggests that the penetration of each injector by FDF approach is much deeper than that of LEM. It is speculated that LEM might provide stronger mixing. In Figure 15, the mass fraction isosurface of the species $\mathrm{OH}$ is shown, and it resembles the temperature structure.
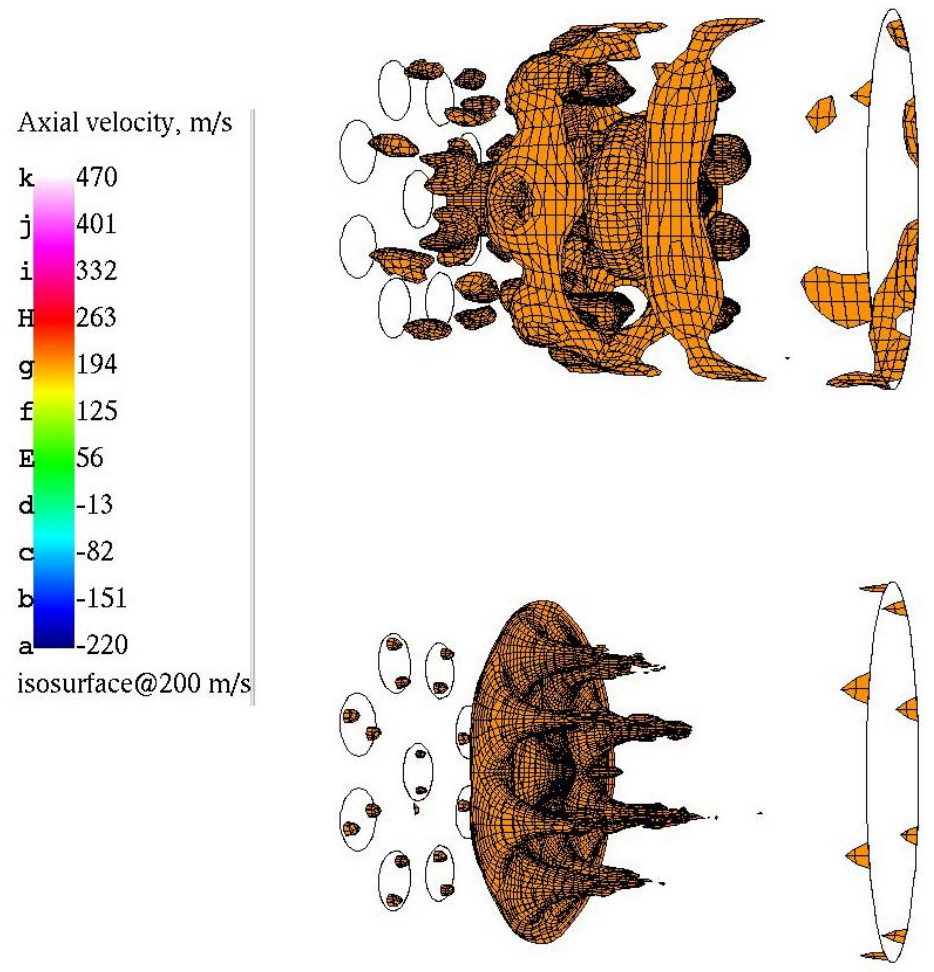

Figure 13. Axial velocity isosurface at the value of $200 \mathrm{~m} / \mathrm{s}$. Top: FDF/PRNS with unsteady pressure exit; Bottom: LEM/PRNS with unsteady pressure exit. 


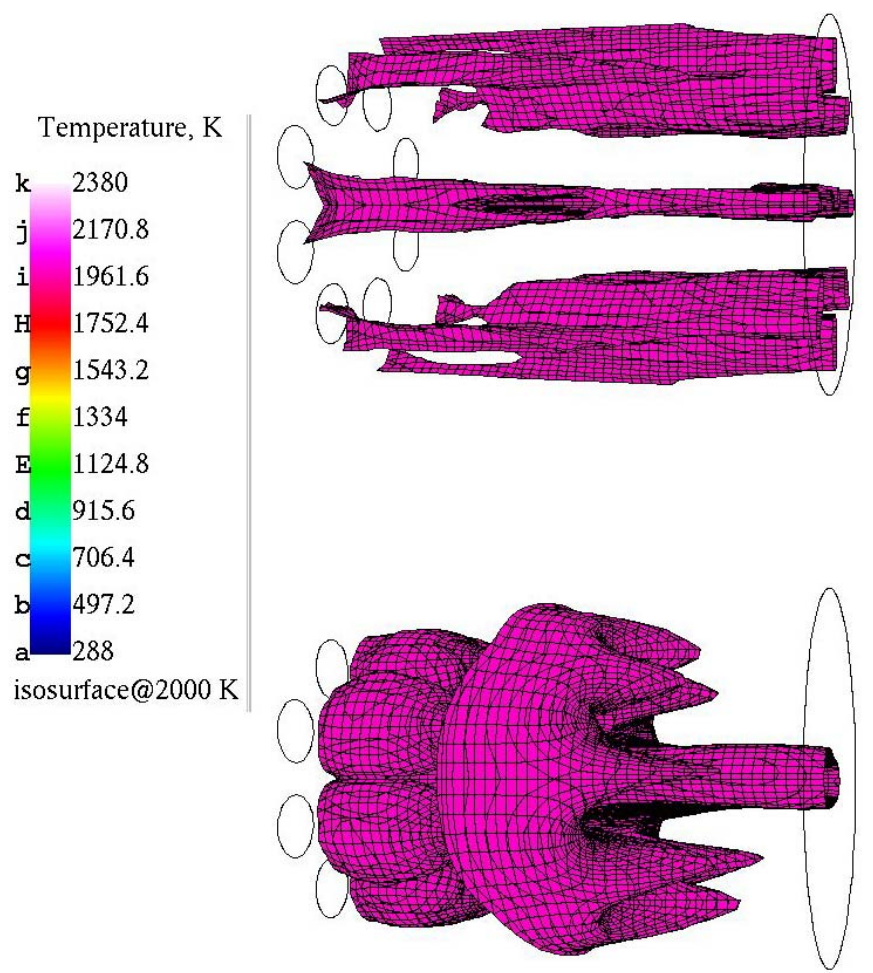

Figure 14. Temperature isosurface at a value of $2000 \mathrm{~K}$. Top: FDF/PRNS with unsteady pressure exit; Bottom: LEM/PRNS with unsteady pressure exit.

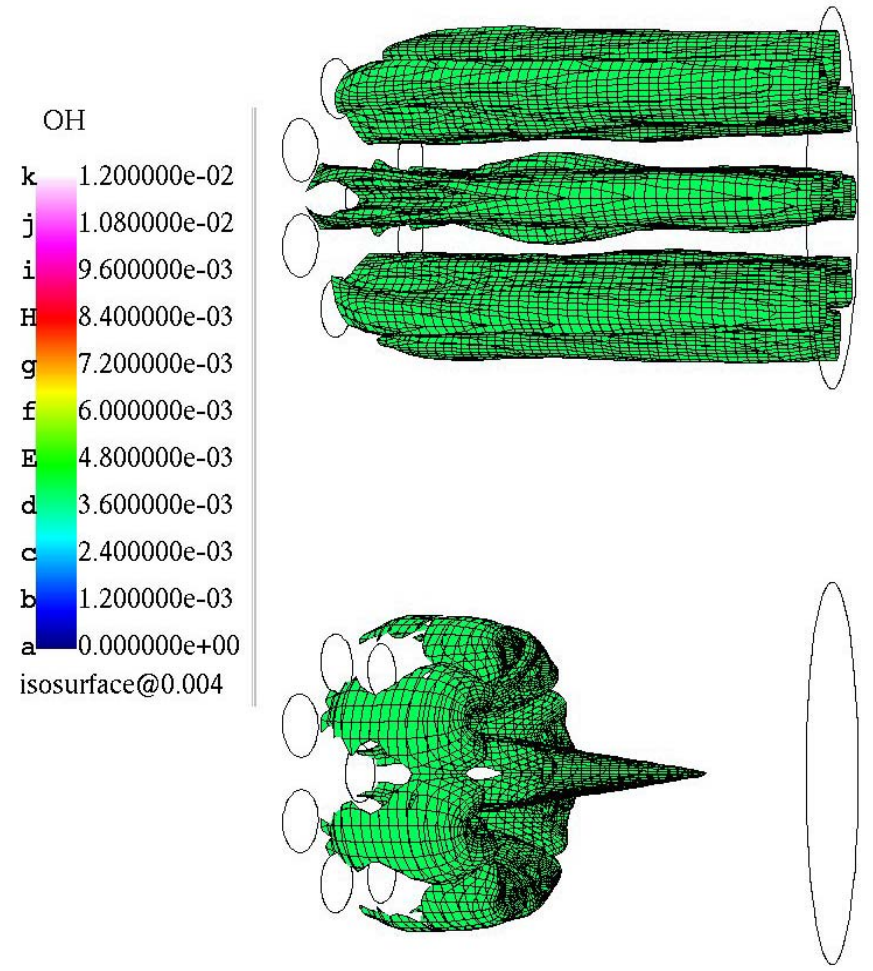

Figure 15. OH mass fraction (.004) isosurface. Top: FDF/PRNS with unsteady pressure exit; Bottom: LEM/PRNS with unsteady pressure exit. 


\section{NASA single-element Jet-A liquid fueled LDI combustor}

Simulations of a single-element lean direct injection (LDI) combustor with liquid spray have been performed. Detailed description of the spray equations and solver can be found in a previous report ${ }^{18}$. The description of the geometry and computational grid has been reported in the literature ${ }^{19}$. Only the boundary conditions for the fuel injector are summarized here. The liquid fuel, $\mathrm{C}_{12} \mathrm{H}_{23}$, is injected into the domain from a pressure swirl injector. The diameter of the orifice is $.0006 \mathrm{~m}$. The fuel atomizer is set at a pressure of $110 \mathrm{kPa}$ and delivers a flow rate of 0.025 $\mathrm{kg} / \mathrm{min}$. The spray has a 90 degree spray angle which is the same as the converging-diverging venture attached to the swirler.

In the laminar chemistry/PRNS case, an inflow droplet size distribution ${ }^{20}$ is prescribed,

$$
\frac{d n}{n}=4.21 \times 10^{6}\left[\frac{d}{d_{32}}\right]^{3.5} e^{-16.98\left(\frac{d}{d_{32}}\right)^{0.4}} \frac{d d}{d_{32}}
$$

where $n$ is the total number of the droplets and $d n$ is the number of droplets in the size range between $d$ and $d+d d$. This correlation also requires the specification of a Sauter mean diameter, $d_{32}$, and the number of droplet classes. These specified inflow droplets will undergo evaporation without secondary breakup.

In the FDF/PRNS case, instead of specifying the inflow droplets, a sheet breakup model ${ }^{21}$ is used for the primary atomization; furthermore, an ETAB (enhanced TAB) mode ${ }^{22}$ is used for the secondary droplet breakup. The value of RCP is set to 0.4 whose selection was discussed in Reference 23. It is also noted here that the solution of the laminar chemistry/PRNS simulation is used as the starting condition for the FDF/PRNS simulation.

Snap shots of instantaneous axial velocity contour at the center plane from PRNS simulations of LDI are shown in Figure 16. The variation of the axial velocity in the case of using FDF is more noticeable than the variation in the case of using laminar chemistry. The temperature results are shown in Figure 17. The irregularity is also more pronounced in the case of using FDF. In Figure18, the sliced contours of the instantaneous temperature at 0.04 meter downstream of the exit of the fuel injector are overlaid by instantaneous temperature isosurfaces at the same location. It can be seen that the results from using the FDF model indicate a more irregular swirling and an earlier merging of the lower temperature portion of the flow.

In the LEM/PRNS case, due to its robustness the prescribed particle size distribution is used as the inflow boundary condition for the fuel injector. For sub-grid scalar field evolution, 18 LEM cells are used within each PRNS cell and up to 40 sub-iteration steps are set for the stirring event and molecular diffusion. The isosurfaces of the mean axial velocity in Figure 19 shows the zone of the recirculation has rather strong reverse flow, about $20 \mathrm{~m} / \mathrm{s}$. In Figure 20 the mean turbulence kinetic energy is mild at the axis near the injector and reduces to smaller values as the flow is marching down stream. The mean temperature at the center plane and several axial stations are shown in Figure 21 and 22 respectively. The influence of the swirler is clearly seen even at the exit plane. In the recirculation zone, the mean temperature at the axis is higher than that of surrounding regions. The equivalence ratio computed from the inlet boundary conditions is about 0.72 . The adiabatic flame temperature is around $2100 \mathrm{~K}$. The mean temperature at the exit plane is between $1800 \mathrm{~K}$ and $1900 \mathrm{~K}$. 


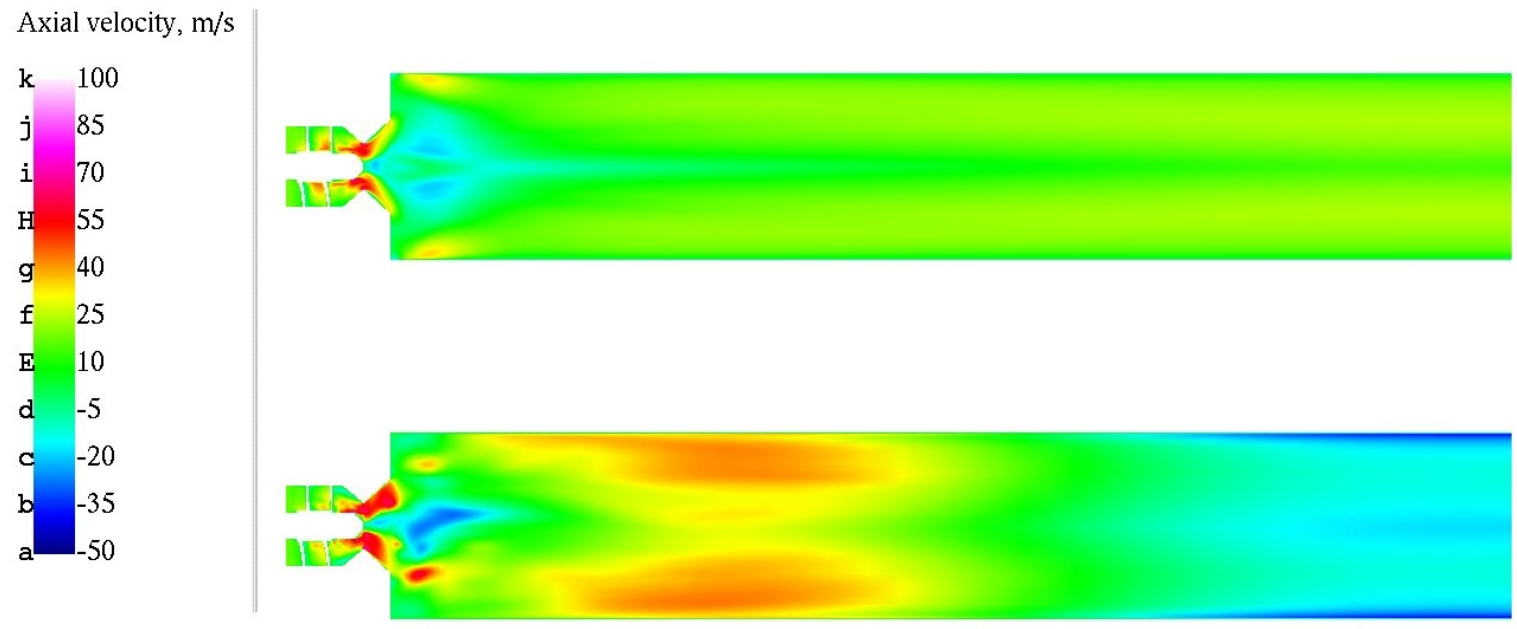

Figure 16. Snap shots of instantaneous axial velocity contour at the center plane from PRNS simulations of LDI: (top) laminar chemistry, (bottom) FDF.

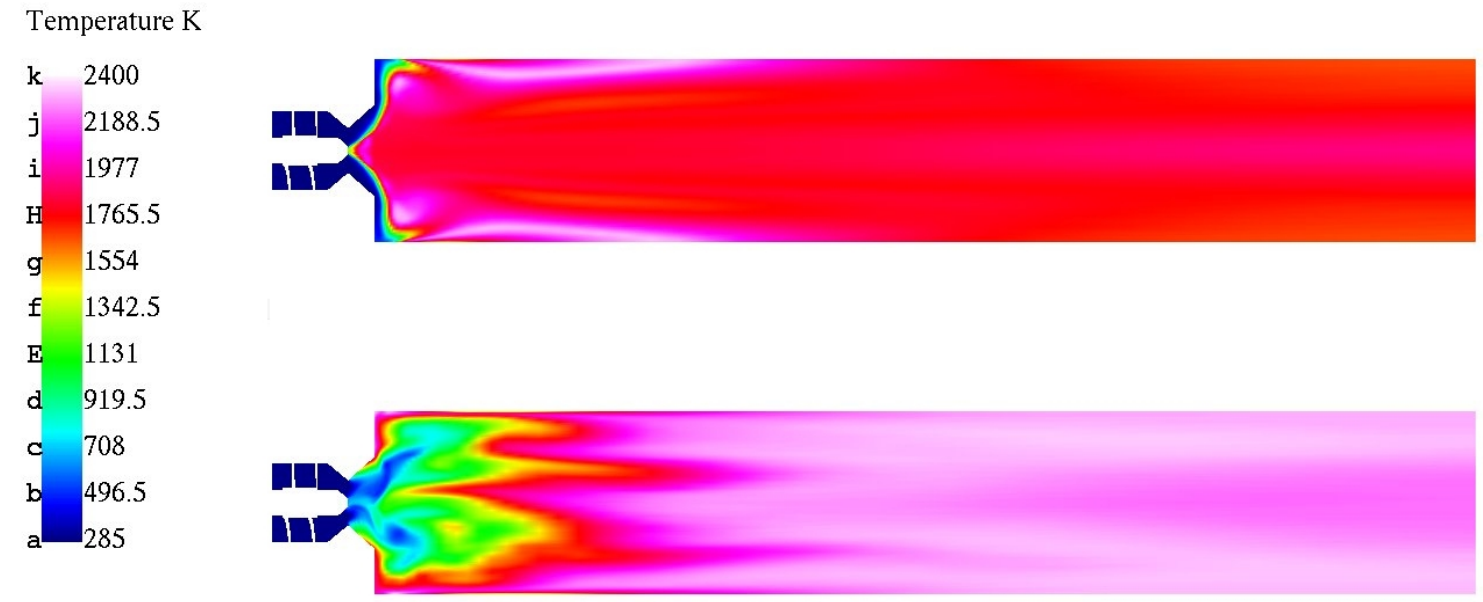

Figure 17. Snap shots of instantaneous temperature contour at the center plane from PRNS simulations of LDI: (top) laminar chemistry, (bottom) FDF.
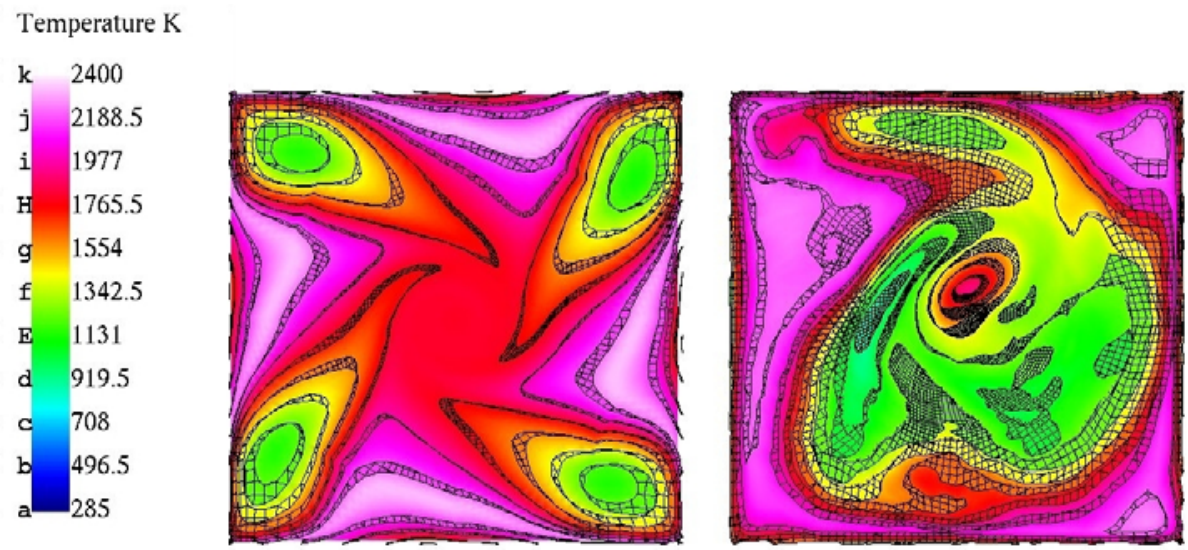

Figure 18. Instantaneous temperature isosurface and sliced contours at $\sim 0.04 \mathrm{~m}$ downstream of the fuel injector (viewed from injector toward exit): left plot from laminar chemistry/PRNS, right plot from FDF/PRNS. 


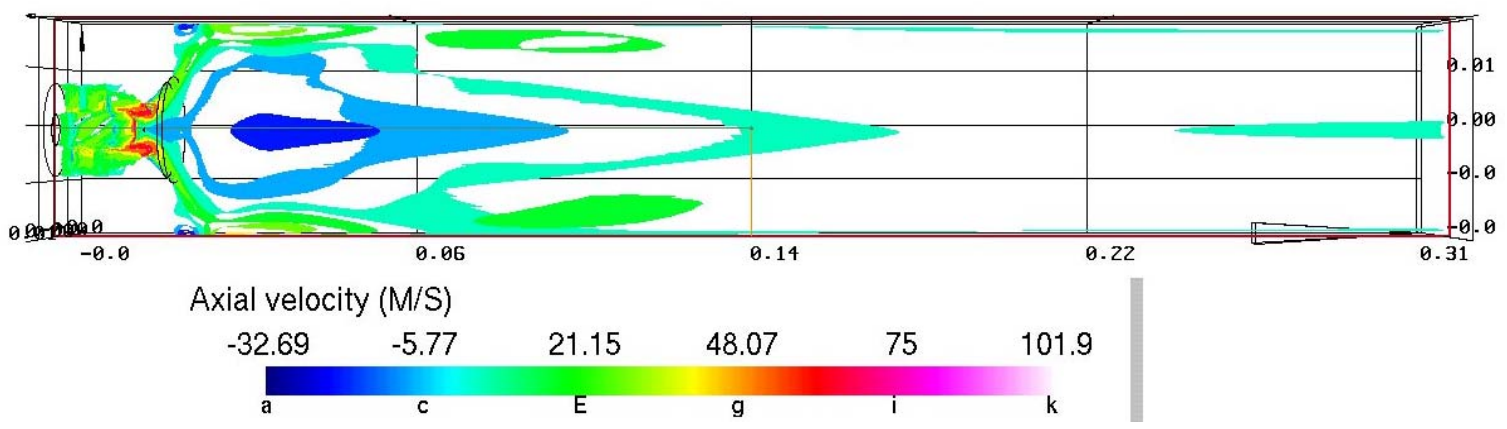

Figure 19. Mean axial velocity isosurface at center plane from PRNS simulations of LEM.

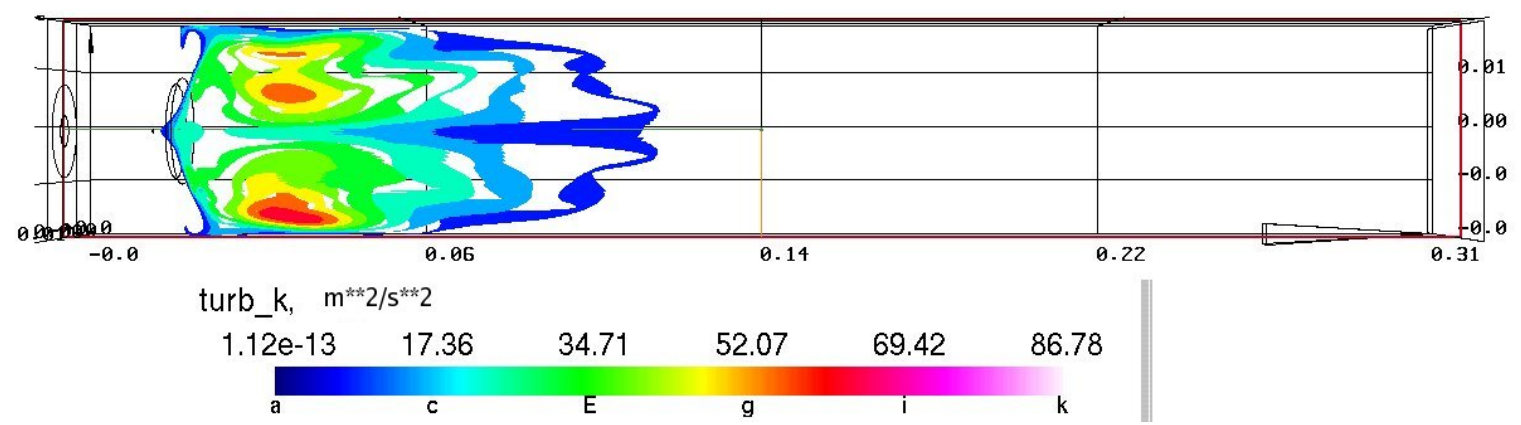

Figure 20. Mean turbulence kinetic energy isosurface at the center plane from PRNS simulations of LEM.

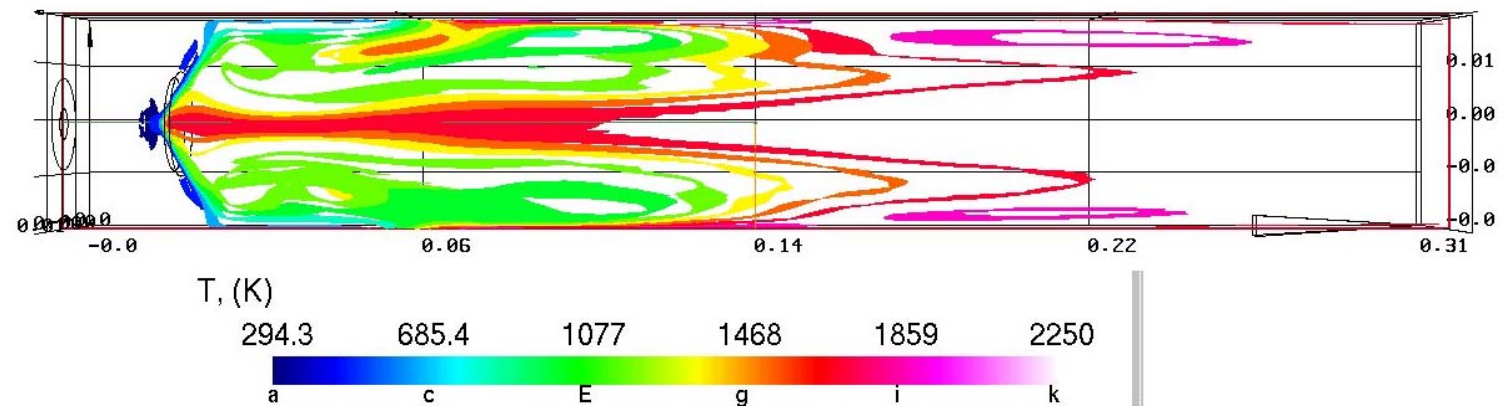

Figure 21. Mean temperature isosurface at center plane from PRNS simulations of LEM.

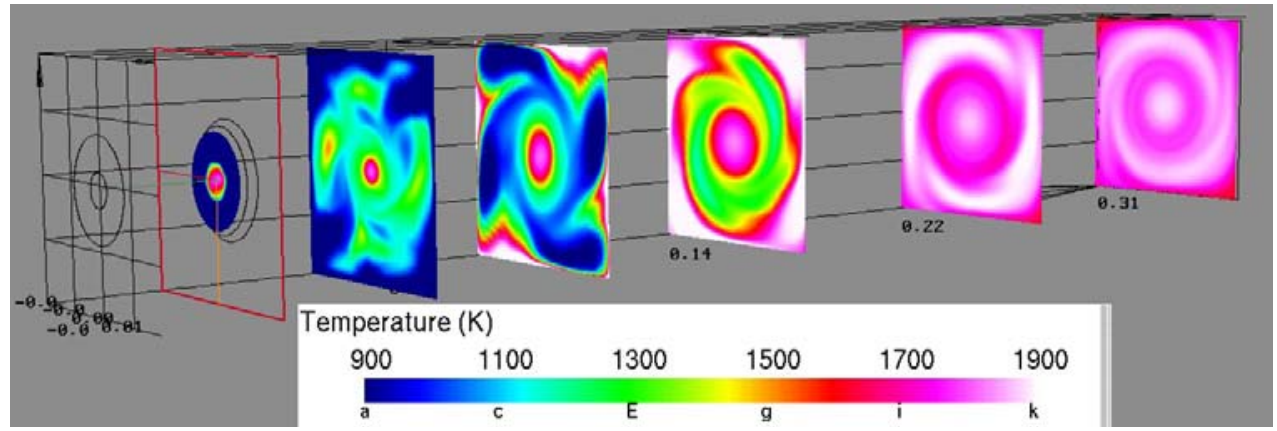

Figure 22. Mean temperature contour plots at six axial stations from PRNS simulations of LEM. 


\section{Concluding Remarks}

Higher fidelity models for turbulence-chemistry interaction are implemented into the NCC. The differences among the LEM, the transport FDF/PDF and the 'laminar chemistry' are examined using the simulation results of a hydrogen gas fueled combustor. Their applications to a Jet-A liquid fueled combustor are also demonstrated. These preliminary results provide the basis for further, quantitative investigations of these higher fidelity models when applied to two-phase turbulent combustion systems. In Part II of the assessment task, the results of the LDI simulations from different turbulent combustion models will be compared with available experimental data.

\section{Acknowledgements}

The authors wish to thank Dr. A. T. Norris who meshed the grid and prepared the inlet boundary conditions for the Sandia 9-element combustor. Dr. Norris also initialized the implementation of LEM into the NCC.

\section{References}

${ }^{1}$ Raju, M. S., “EUPDF-II: An Eulerian Joint Scalar Monte Carlo PDF Module - User’s Manual,” NASA CR 2004-213073.

${ }^{2}$ Menon, S., Sankaran, V., and Stone, C., "Subgrid Combustion Modeling for the Next Generation National Combustion Code," NASA CR 2003-212202.

${ }^{3}$ Colucci, P. J., Jaberi, F. A., Givi, P. and Pope, S. B., “ Filtered Density function for Large Eddy Simulation of Turbulent Reacting Flows,” Phys. Fluid Vol. 10, pp. 499-515, 1998.

${ }^{4}$ Pope, S. B., “Turbulent Flows,” Cambridge University Press, 771pp, pp. 59.

${ }^{5}$ Lundgren, T. S., “Distribution Functions in the Statistical Theory of Turbulence,” Phys. Fluids, Vol. 10, pp.969-975.

${ }^{6}$ Lundgren, T. S., “Model Equation for Nonhomogeneous Turbulence,” Phys. Fluids, Vol. 12, pp.485-497.

${ }^{7}$ Wey, T. C. and Liu, N.-S., "Current Status of Post-Combustor Trace Chemistry Modeling and Simulation at NASA Glenn Research Center,” NASA/TM---2003-212184, March 2003.

${ }^{8}$ Kerstein, A. R., "Linear-Eddy Model of Turbulent Transport. 2. Application of Shear-Layer Mizing," Combustion and Flame 75 (1989) 397-413.

${ }^{9}$ Kerstein, A. R., "Linear-Eddy Model of Turbulent Transport. Part 4. Structure of Diffusion Flames," Combustion Science and Technology 81 (1992) 75-96.

${ }^{10}$ Chakravarthy, V. K. and Menon, S., “Modeling of Turbulent Premixed Flames in the Flamelet Regime,” Combustion, Science and Technology, Vol. 162, pp.1-50.

${ }^{11}$ Chakravarthy, V. K. and Menon S., "Subgrid Modeling of Turbulent Premixed Flames in the Flamelet Regime,” Flw, Turbulence and Combustion, Vol 65.

${ }^{12}$ Pope, S. B., “PDF Methods for Turbulent Reactive Flow,” Prg. Energy Combust. Sci., Vol. 11, pp. 119-192, 1985.

${ }^{13}$ Chen, J.-Y., "A Eulerian PDF Scheme for LES of Nonpremixed Turbulent Combustion with Second-order accurate mixture fraction,” Combustion Theory and Modeling, Vol. 11, No. 5, October 2007, pp. 675-695.

${ }^{14}$ Borghi, R., “Turbulent Combustion Modeling,” Prog. Energy Combust. Sci., Vol. 14 pp. 245-292, 1988

${ }^{15}$ Shih, T.-H. and Liu, N.-S., “Assessment of Partially Resolved Numerical Simulation (PRNS) Approach in the National Combustion Code (NCC) for Turbulent Non-reacting and Reacting Flow, ” NASA TM-2008-215418.

${ }^{16}$ Liu, N.-S. and Shih, T.-H., "Turbulence Modeling for Very Large-Eddy Simulation,” AIAA Journal, Vol. 44, No. 4, 2006, pp.687-697.

${ }^{17}$ Shih, T.-H. and Liu, N.-S., "Numerical Study of Outlet Boundary Conditions for Unsteady Turbulent Internal Flows Using the NCC,” NASA TM 2009-215486.

${ }^{18}$ Raju, M. S., “LSPRAY-III: A Lagrangian Spray Module,” NASA/CR—2008-215290, July 2008.

${ }^{19}$ Davoudzadeh, F., Liu, N.-S. and Moder, J. P., "Investigation of Swirling Air Flows Generated by Axial Swirler in a Flame Tube,” Proceedings of GT2006 ASME Turbo Expo 2006: Power for Land, Sea and Air May 8-11, 2006, Barcelona, Spain.

${ }^{20}$ El Banhawy, Y. and Whitelaw, J. H., "Calculation of the Flow Properties of a Confined Kerosene-Spray Flame,” AIAA J., Vol. 18, No. 12, pp.1503-1510, 1980.

${ }^{21}$ Schmidt, D. P., Nouar, I. Senecal, P. K., Hoffman, J., Rutland, C. J., Martin, J. and Reitz, R. D., "Pressure-Swirl Atomization in the Near Field,” 1999 SAE Congress, SAE 1999-01-0496.

${ }^{22}$ Tanner, F. X., “A Cascade Atomization and Drop Breakup Model for the Simulation of High-Pressure Liquid Jets,” SAE Paper 2003-01-1044, 2003.

${ }^{23}$ Shih, T.-H. and Liu, N.-S., “A Nonlinear Dynamic Subscale Model for PRNS/VLES of Internal Combustor Flows,” AIAA 2009-0467, Jan. 4-8, 2009, Orlando, FL. 